\title{
Dielectric relaxation in double perovskite oxide, $\mathrm{Ho}_{2} \mathrm{CdTiO}_{6}$
}

\author{
DEV K MAHATO*, A DUTTA ${ }^{\dagger}$ and T P SINHA ${ }^{\dagger}$ \\ Department of Physics, National Institute of Technology Patna, Ashok Rajpath, Patna 800 005, India \\ $\dagger$ Department of Physics, Bose Institute, 93/1, Acharya Prafulla Chandra Road, Kolkata 700 009, India
}

MS received 9 August 2010; revised 16 November 2010

\begin{abstract}
A new double perovskite oxide holmium cadmium titanate, $\mathrm{Ho}_{2} \mathrm{CdTiO}_{6}$ (HCT), prepared by solid state reaction technique is investigated by impedance spectroscopy in a temperature range $50-400^{\circ} \mathrm{C}$ and a frequency range 75 Hz-1 MHz. The crystal structure has been determined by powder X-ray diffraction which shows monoclinic phase at room temperature. An analysis of complex permittivity with frequency was carried out assuming a distribution of relaxation times as confirmed by Cole-Cole plot. The frequency dependent electrical data are analysed in the framework of conductivity and electric modulus formalisms. The frequencies corresponding to the maxima of the imaginary electric modulus at various temperatures are found to obey an Arrhenius law with an activation energy of $0.13 \mathrm{eV}$. The scaling behaviour of imaginary part of electric modulus suggests that the relaxation describes the same mechanism at various temperatures. Nyquist plots are drawn to identify an equivalent circuit and to know the bulk and interface contributions.
\end{abstract}

Keywords. Holmium cadmium titanate; double perovskite; dielectric relaxation; impedance spectroscopy.

\section{Introduction}

Perovskites are an important large class of compounds with a stoichiometry, $\mathrm{ABO}_{3}$, and have been studied extensively due not only to the versatile nature of the structure but more importantly for their many useful properties. Perovskites exhibit magnetic (e.g. $\mathrm{La}_{1-x} \mathrm{~B}_{x}^{2+} \mathrm{MnO}_{3}$ ) (Goodenough 1955), dielectric $\left(\mathrm{BaTiO}_{3}\right)$ (Bidault et al 1994), high temperature superconducting $\left(\mathrm{BaPb}_{1-x} \mathrm{Bi}_{x} \mathrm{O}_{3}\right)$ (Claeson et al 1989; Sleight et al 1993), piezoelectric $\left(\mathrm{PbZr}_{1-x} \mathrm{Ti}_{x} \mathrm{O}_{3}\right)$ (Lucuta et al 1985), and ferroelectric properties $\left(\mathrm{KNbO}_{3}\right)$ (Hewat 1973). The ideal ternary perovskite structure has the aforementioned $\mathrm{ABO}_{3}$ stoichiometry, where $A$ is typically a large, low oxidation state cation, $B$ a smaller transition metal or lanthanide cation and adopts cubic symmetry (Mitchell 2002). B-site cations are octahedrally coordinated to six $O$-anions, and $A$-site cations are cubooctahedrally coordinated to twelve $O$-anions. The $A$-site cation sits in the body centre of the cube formed by eight corner sharing $\mathrm{BO}_{6}$ octahedra. In complex perovskite, two or more cations of different valences are located in equivalent crystallographic positions. For such systems when there is a charge and/or size difference between the $B$ cations an ordering can occur. This ordering often results in the double perovskite structure with general stoichiometry $\mathrm{A}_{2} \mathrm{~B}^{\prime} \mathrm{B}^{\prime \prime} \mathrm{O}_{6}$.

Double perovskite oxide with general formula, $A_{2} B^{\prime} B^{\prime \prime} O_{6}$ unit structure, has been widely studied due to its surprising transport properties (Kobayashi et al 1998; Zhao et al 2005;

*Author for correspondence (drdevkumar@yahoo.com)
Vijaykumar et al 2009; Kumar et al 2009; Dutta et al 2010). It has been the subject of recent investigation owing to its inherent ability to accommodate a wide range of elemental compositions exhibiting a range of properties including dielectric, piezo-, ferroelectric, optical, superconducting and magnetoresistive properties (Raveau 2007) and to display a wealth of structure variants (Saines et al 2007; Bharti and Sinha 2010).

Extensive studies relating to different aspects of these double perovskites have been made especially for their potential applications arising from their interesting electrical and magnetic properties (Kobayashi et al 1998; DeMarco 2000). Anderson et al (1993) have shown that ordered structure is most likely to be formed if charge difference between the two $B$-site cations is two or higher. Due to the complicated characteristics of double perovskite oxide and nonavailability of uniform explanation of its various properties, $A_{2} B^{\prime} B^{\prime \prime} O_{6}$ compounds have become very exciting materials for researchers.

In the present investigation, we have prepared a new double perovskite oxide $\mathrm{Ho}_{2} \mathrm{CdTiO}_{6}$ (HCT) by solid state reaction technique and studied its dielectric relaxation behaviour in a wide range of frequency $(75 \mathrm{~Hz}-1 \mathrm{MHz})$ and temperature $\left(50-400^{\circ} \mathrm{C}\right)$ by impedance spectroscopy.

\section{Experimental}

The complex perovskite oxide of $\mathrm{Ho}_{2} \mathrm{CdTiO}_{6}$ (HCT) was synthesized by solid state reaction technique. Powders of 


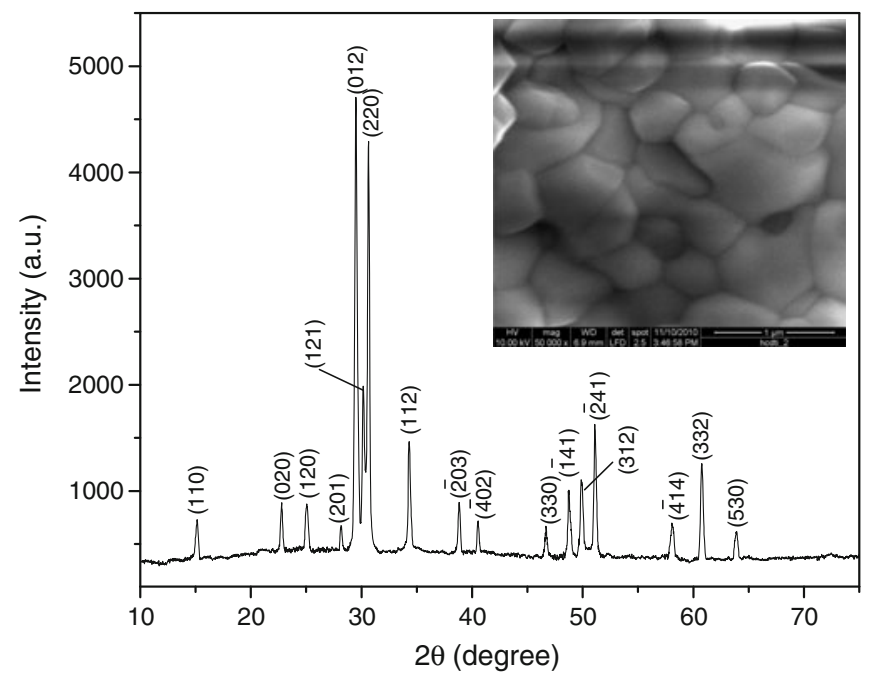

Figure 1. XRD pattern of $\mathrm{Ho}_{2} \mathrm{CdTiO}_{6}$ at room temperature.

$\mathrm{Ho}_{2} \mathrm{O}_{3}$ (Aldrich, 99.9+\%), $\mathrm{CdO}$ (Loba Chemie, 99.5\%) and $\mathrm{TiO}_{2}$ (Laboratory reagent, $98 \%$ ) were taken as primary raw materials and mixed in stoichiometric ratio in the presence of acetone (MERCK) for $12 \mathrm{~h}$. The mixture was calcined in a Pt crucible at $1200^{\circ} \mathrm{C}$ in air for $10 \mathrm{~h}$ and brought to room temperature at a cooling rate of $100^{\circ} \mathrm{C} / \mathrm{h}$. The calcined powder was analysed by X-ray diffraction technique and it was found to have monoclinic structure. X-ray diffractogram on calcined powder of HCT was recorded at room temperature in a wide range of Bragg angles $\left(10^{\circ} \leq 2 \theta \leq 80^{\circ}\right)$, using $\mathrm{CuK}_{\alpha}$ radiation $(\lambda=1.5418 \AA)$ and a $\mathrm{Ni}$ filter operating at $40 \mathrm{kV}$ and $20 \mathrm{~mA}$ with a scanning rate of $2 \%$ min by a powder X-ray diffractometer (Rigaku Miniflex-II). The scanning electron micrograph (SEM) of the sample is taken by a FEI Quanta 200 scanning electron microscope. The calcined sample was pelletized into a circular disc (of thickness $2.2 \mathrm{~mm}$ and dia. $4.5 \mathrm{~mm}$ ) using PVA as binder which was burnt out during high temperature sintering at $1250^{\circ} \mathrm{C}$ for $5 \mathrm{~h}$, and cooled slowly to room temperature by adjusting the cooling rate. The sintered pellets were polished to make both their faces parallel and electroded by high purity ultrafine silver paste for their electrical characterization. In order to dry the paste, samples were evaporated onto both sides of the disc at $200^{\circ} \mathrm{C}$ for $2 \mathrm{~h}$ prior to conducting experiment. Capacitance $(C)$, impedance $(Z)$, phase angle $(\phi)$ and conductance $(G)$, of the sample were measured both as a function of frequency $(75 \mathrm{~Hz}-1 \mathrm{MHz})$ and temperature $\left(50-400^{\circ} \mathrm{C}\right)$ using a computer controlled LCR-meter (HIOKI-3552, Japan). The temperature was controlled with a programmable oven. Each measured temperature was kept constant with an accuracy of $\pm 1 \mathrm{~K}$.

\section{Results and discussion}

The X-ray diffraction pattern of HCT measured at room temperature is shown in figure 1 . All the reflection peaks of the X-ray profile are indexed and lattice parameters are determined using a least-squares method with the help of a standard computer programme (Crysfire). Good agreement between the observed and calculated interplanner spacings ( $d$-values) suggests that the compound crystallizes in monoclinic phase at room temperature with cell parameters $a=$ 9.3858, $b=7.8034, c=7.0387$. X-ray diffraction confirms

Table 1. Comparison of observed and calculated $d$-values $\AA$ with reflections of $\mathrm{Ho}_{2} \mathrm{CdTiO}_{6}$ at room temperature.

\begin{tabular}{|c|c|c|c|c|}
\hline $2 \theta$ (degree) & $h k l$ & $\begin{array}{l}d \text {-spacing } \AA \\
\text { observed (a) }\end{array}$ & $\begin{array}{c}d \text {-spacing } \AA \\
\text { calculated (b) }\end{array}$ & $\begin{array}{l}\text { Difference } \\
\quad(a-b)\end{array}$ \\
\hline $15 \cdot 178$ & 110 & 5.8329 & $5 \cdot 8262$ & $0 \cdot 0067$ \\
\hline $22 \cdot 760$ & 020 & 3.9039 & 3.9002 & 0.0037 \\
\hline 24.960 & 120 & $3 \cdot 5646$ & $3 \cdot 5637$ & 0.0009 \\
\hline $28 \cdot 160$ & 201 & $3 \cdot 1664$ & $3 \cdot 1675$ & $-0 \cdot 0011$ \\
\hline 29.460 & 012 & 3.0295 & 3.0308 & -0.0013 \\
\hline $30 \cdot 140$ & 121 & 2.9627 & 2.9602 & $0 \cdot 0025$ \\
\hline $30 \cdot 620$ & 220 & $2 \cdot 9173$ & 2.9146 & 0.0033 \\
\hline $34 \cdot 280$ & 112 & 2.6138 & 2.6133 & 0.0005 \\
\hline $38 \cdot 800$ & $\overline{2} 03$ & $2 \cdot 3191$ & $2 \cdot 3175$ & 0.0016 \\
\hline $40 \cdot 500$ & $\overline{4} 20$ & $2 \cdot 2255$ & $2 \cdot 2249$ & 0.0006 \\
\hline $46 \cdot 680$ & 330 & 1.9443 & 1.9431 & 0.0012 \\
\hline $48 \cdot 720$ & $\overline{1} 41$ & 1.8675 & 1.8661 & 0.0014 \\
\hline $49 \cdot 840$ & 312 & $1 \cdot 8282$ & $1 \cdot 8271$ & $0 \cdot 0011$ \\
\hline $51 \cdot 080$ & $\overline{2} 41$ & 1.7867 & 1.7861 & 0.0006 \\
\hline 58.080 & $\overline{4} 14$ & 1.5869 & 1.5863 & 0.0006 \\
\hline $60 \cdot 740$ & 332 & 1.5236 & 1.5231 & 0.0005 \\
\hline 63.940 & 530 & 1.4548 & 1.4543 & 0.0005 \\
\hline
\end{tabular}




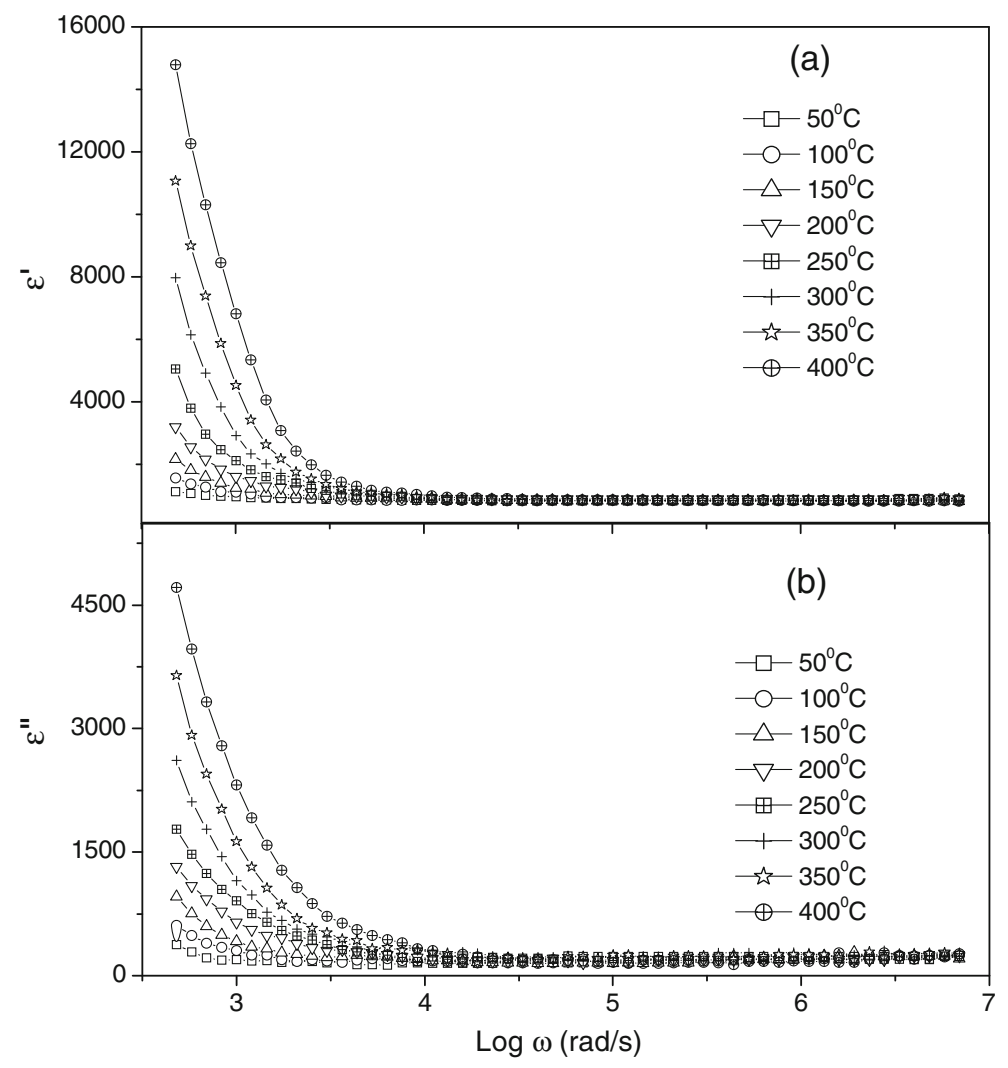

Figure 2. Frequency dependence of (a) $\varepsilon^{\prime}$ and (b) $\varepsilon^{\prime \prime}$ of $\mathrm{Ho}_{2} \mathrm{CdTiO}_{6}$ at various temperatures.

that the specimen is single phase. The observed and calculated $d$-values are listed in table 1. The SEM image of HCT as shown in the inset of figure 1 indicates the high density of the material as well as a uniform distribution of grains of different sizes and shapes. The average grain size of HCT is $0.4 \mu \mathrm{m}$.

The angular frequency, $\omega(=2 \pi \nu)$, dependence of the dielectric constant $\left(\varepsilon^{\prime}\right)$ of HCT as a function of temperature is shown in figure $2 \mathrm{a}$. The nature of dielectric permittivity related to free dipoles oscillating in an alternating field may be described as follows. When the frequency of alternating field is much less than the inverse of relaxation time $(\tau)$ (i.e. $\omega \ll 1 / \tau)$, electric dipoles follow the field and we have $\varepsilon^{\prime} \approx$ $\varepsilon_{\mathrm{s}}\left(\varepsilon_{\mathrm{s}}=\right.$ low frequency value of $\varepsilon^{\prime}$ or value of dielectric constant at quasistatic fields). As the frequency increases $(\omega<$ $1 / \tau)$, dipoles start to lag behind the field and dielectric constant $\left(\varepsilon^{\prime}\right)$ slightly decreases. When frequency becomes the characteristic frequency $(\omega=1 / \tau)$, $\varepsilon^{\prime}$ drops suddenly indicating relaxation process. At very high frequency $(\omega \gg 1 / \tau)$, dipoles can no longer follow the field and we have $\varepsilon^{\prime} \approx$ $\varepsilon_{\infty}\left(\varepsilon_{\infty}=\right.$ high frequency value of $\left.\varepsilon^{\prime}\right)$. This qualitative behaviour may be observed in figure $2 \mathrm{a}$. The dielectric constant gradually decreases with increasing frequency and becomes almost frequency independent at higher frequency region. With increase in temperature, it increases apparently and becomes even more significant at low frequency range.

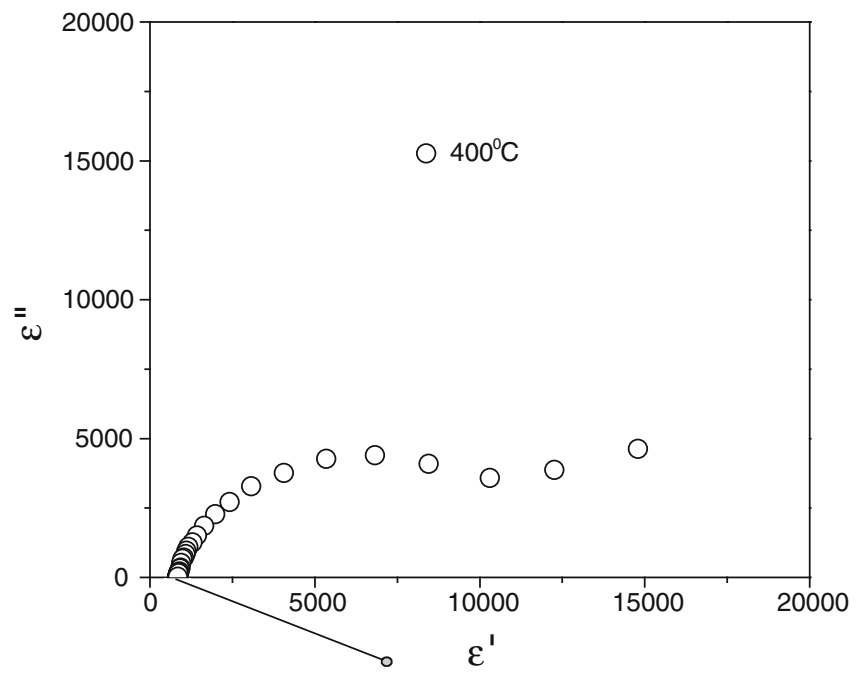

Figure 3. Complex Argand plane plot between $\varepsilon^{\prime}$ and $\varepsilon^{\prime \prime}$ for $\mathrm{Ho}_{2} \mathrm{CdTiO}_{6}$ at $400^{\circ} \mathrm{C}$.

Figure $2 \mathrm{~b}$ shows the angular frequency $(\omega)$ dependence of dielectric loss $\left(\varepsilon^{\prime \prime}\right)$ of HCT as a function of temperature. Similar trends of $\varepsilon^{\prime \prime}$ as a function of frequency and temperature is observed in the case of $\varepsilon^{\prime}$ (figure 2a). At lower frequency range, dielectric loss is rather high for all 
temperatures, and decreases with increasing frequency and it increases with increasing temperature similar to the dependence of dielectric constant on temperature. This indicates the thermally activated nature of the dielectric relaxation

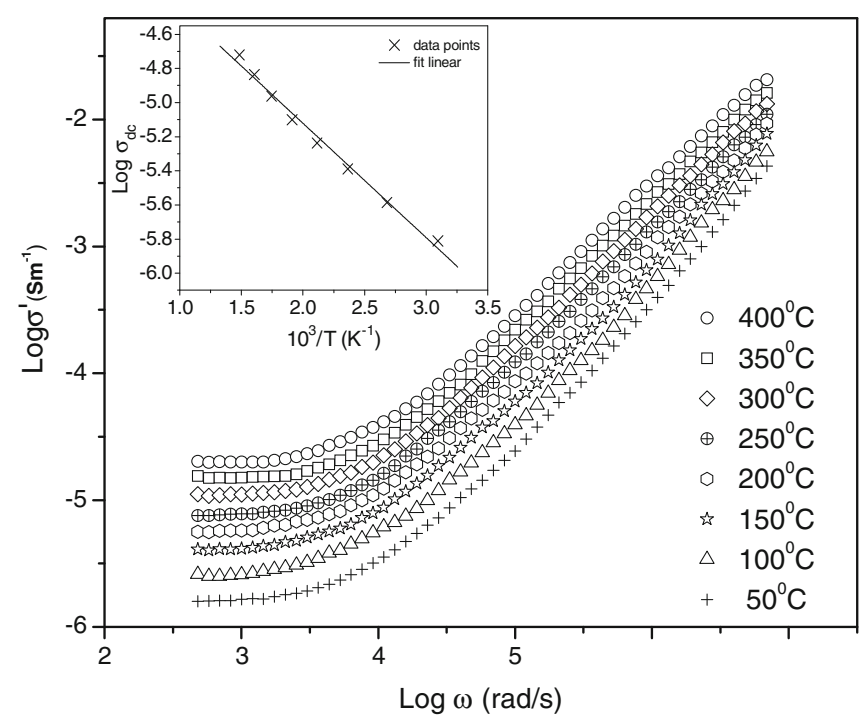

Figure 4. Frequency dependence of conductivity $\left(\sigma^{\prime}\right)$ for $\mathrm{Ho}_{2} \mathrm{CdTiO}_{6}$ at various temperatures. The temperature dependence of d.c. conductivity curve for $\mathrm{Ho}_{2} \mathrm{CdTiO}_{6}$ is shown in the inset where the symbols are the experimental points and the solid line is the least-squares straight line fit. of the HCT system. The fast rising trend of $\varepsilon^{\prime \prime}$ at low frequencies is due to the presence of d.c. conductivity in HCT.

In the analysis of dielectric relaxation in the frequency domain, a very convenient representation, in terms of the Argand diagram of the complex plane of the relaxation function was introduced by Cole and Cole (1941). Figure 3 shows a representative complex Argand plane plots between $\varepsilon^{\prime \prime}$ and $\varepsilon^{\prime}$ for $T=400^{\circ} \mathrm{C}$. For a pure monodispersive Debye process, one expects semicircular plots with a centre located on the $\varepsilon^{\prime}$ axis, whereas for polydispersive relaxation, these Argand plane plots are close to circular arcs with end points on the axis of real and a centre below this axis. The complex dielectric constant in such situations is known to be described by the empirical relation

$$
\varepsilon^{*}=\varepsilon_{\infty}+\frac{\left(\varepsilon_{\mathrm{s}}-\varepsilon_{\infty}\right)}{1+(j \omega \tau)^{1-\alpha}},
$$

where $\alpha$ is a measure of the distribution of relaxation times and is zero for monodispersive Debye process. The parameter, $\alpha$, can be determined from the angle subtended by the radius of Cole-Cole circle with the $\varepsilon^{\prime}$-axis passing through the origin of the $\varepsilon^{\prime \prime}$-axis and is found to be 0.51 at $400^{\circ} \mathrm{C}$. The Cole-Cole plot confirms the polydispersive nature of dielectric relaxation of HCT.

It may be noted that the high values of $\varepsilon^{\prime}$ and $\varepsilon^{\prime \prime}$ at frequencies lower than about $1 \mathrm{kHz}$ do not generally correspond to bulk effect. The high values of $\varepsilon^{\prime}$ interestingly observed only at very high temperature and very low frequencies may be

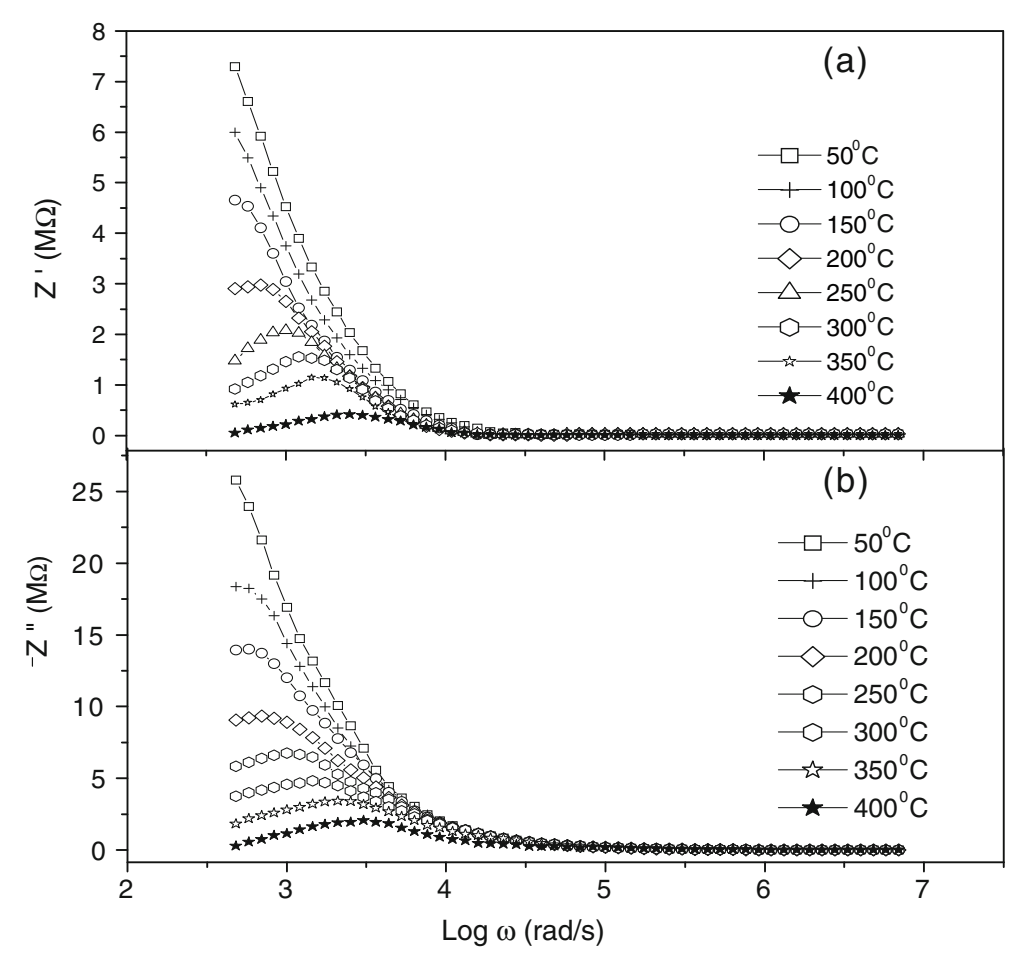

Figure 5. Frequency dependence of (a) $Z^{\prime}$ and (b) $Z^{\prime \prime}$ for $\mathrm{Ho}_{2} \mathrm{CdTiO}_{6}$ at various temperatures. 
attributed to the fact that the free charges buildup at interfaces within the bulk of the sample (interfacial Maxwell-Wagner (MW) polarization) (Kyritsis et al 1995) and at the interface between the sample and the electrodes space-change polarization (Gerhardt 1994). In order to elucidate this point, the frequency dependent a.c. conductivity at various temperatures is plotted in figure 4. The frequency dependent a.c. conductivity is given by the relation

$$
\sigma_{\mathrm{ac}}=\varepsilon_{0} \omega \varepsilon^{\prime \prime} .
$$

A plateau region (where $\sigma_{\mathrm{ac}}$ is frequency independent) is observed in the plots which extends to higher frequencies with increasing temperature. It is the region of d.c. conductivity $\left(\sigma_{\mathrm{dc}}\right)$. The value of $\sigma_{\mathrm{ac}}$ decreases with decreasing frequency as shown in figure 4. At very low frequencies and high temperatures, this drop co-relates quite well with the increase in $\varepsilon^{\prime}$ as shown in figure 2. The values of $\sigma_{\mathrm{dc}}$ obtained from low-frequency plateau follow Arrhenius law given by

$$
\sigma_{\mathrm{dc}}=\sigma_{0} \exp \frac{-E_{\sigma}}{k_{\beta} T},
$$

with an activation energy $0 \cdot 13 \mathrm{eV}$ as shown in the inset of figure 4 .

In order to study the relaxation mechanism in HCT, the impedance and modulus formalism has been adopted in this work. In the spectroscopic impedance, $Z^{*}$ and electric modulus, $M^{*}$ analyses, the imaginary impedance, $Z^{\prime \prime}$ and modulus, $M^{\prime \prime}$ are plotted as a function of frequency, respectively. The peak is observed in these plots corresponding to a relaxation process. The peak height in $Z^{\prime \prime}$ vs frequency plot is proportional to the resistance of that process, while the peak height in $M^{\prime \prime}$ versus frequency plot is inversely proportional to the capacitance. The peak position in each of these plots correspond to the condition $\omega_{\mathrm{m}} \tau_{\mathrm{m}}=1$.

Figure 5 shows the angular frequency dependence of $Z^{\prime}(\omega)$ and $Z^{\prime \prime}(\omega)$ of HCT as a function of temperature. In figure $5(a)$, the variation of $Z^{\prime}$ with frequency for all temperatures implies relaxation process in the sample. The magnitude of $Z^{\prime}$ decreases with increasing temperature at lower frequency which indicates increase in the a.c. conductivity in HCT. The $Z^{\prime}$ values merge at higher frequencies (above $2 \mathrm{kHz}$ ) for all temperatures indicating release of space charges. From figure 5 (b) it is clear that the position of the peak in $Z^{\prime \prime}$ (centred at the dispersion region of $Z^{\prime}$ ) shifts to higher frequencies with increase in temperature and a strong dispersion of $Z^{\prime \prime}$ exists. The width of the peak in figure 5(b) points towards the possibility of a distribution of relaxation times. In such a situation, the most probable relaxation time $\tau_{m}\left(=1 / \omega_{\mathrm{m}}\right)$ from the position of the peak in the $Z^{\prime \prime}$ versus $\log \omega$ plots can be determined. The most probable relaxation time follows the Arrhenius law given by the relation

$$
\omega_{\mathrm{m}}=\omega_{0} \exp \left(\frac{-E_{\mathrm{a}}}{k_{\mathrm{B}} T}\right),
$$

where $\omega_{0}$ is the pre-exponential factor and $E_{\mathrm{a}}$ the activation energy.

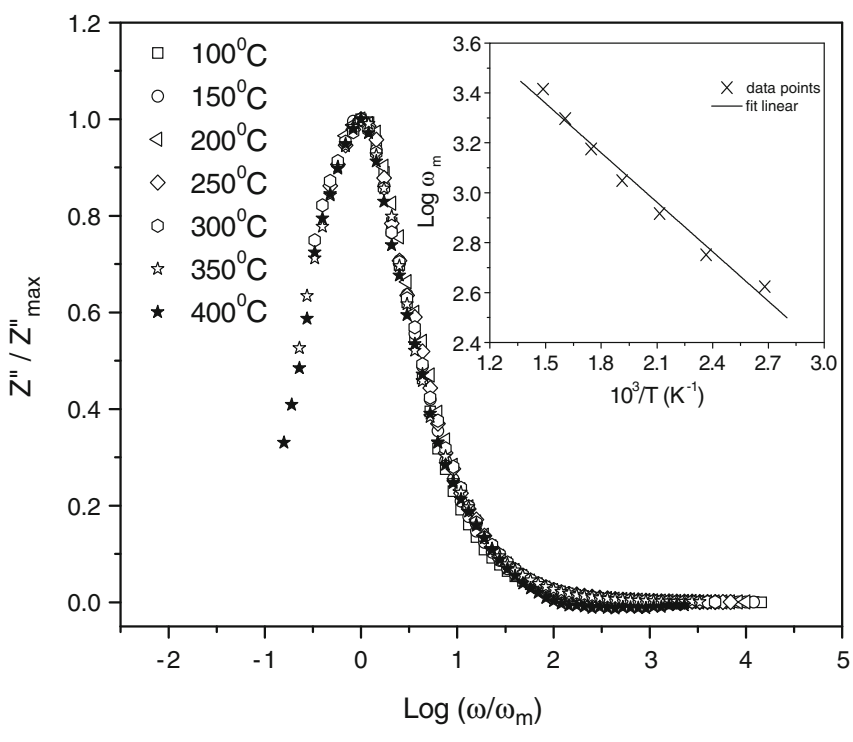

Figure 6. Scaling behaviour of $Z^{\prime \prime}$ at various temperatures for $\mathrm{Ho}_{2} \mathrm{CdTiO}_{6}$. The temperature dependence of the most probable relaxation frequency obtained from the frequency-dependent plot of $Z^{\prime \prime}$ is shown in the inset where the symbols are the experimental points and the solid line is the least-squares straight line fit.

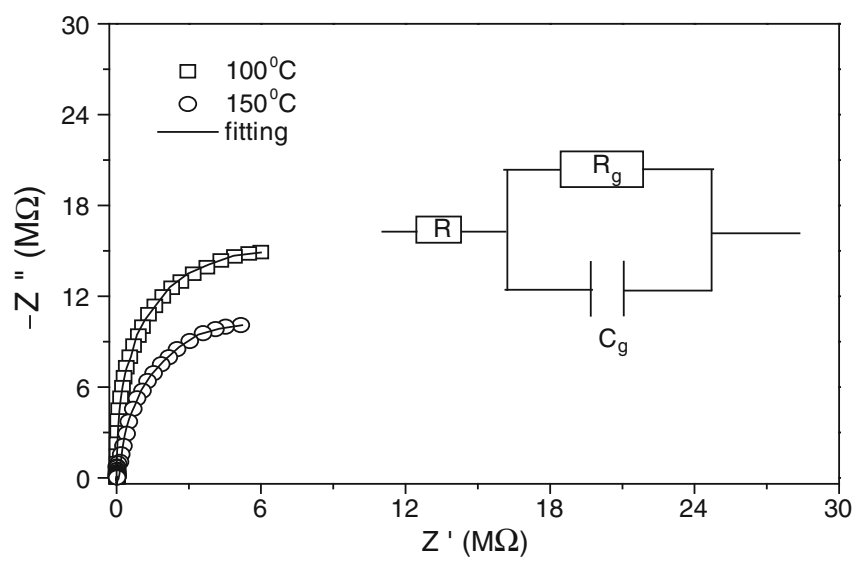

Figure 7. Complex impedance plane plot for $\mathrm{Ho}_{2} \mathrm{CdTiO}_{6}$ at $100^{\circ} \mathrm{C}$ and $150^{\circ} \mathrm{C}$ where solid lines are fitting to experimental data by $\mathrm{RC}$ equivalent circuit.

A plot of $\log \omega_{\mathrm{m}}$ versus inverse of absolute temperature $(1 / T)$ is shown in the inset of figure 6 , where the symbols are the experimental data and the solid line is the least-squares straight-line fit. The activation energy, $E_{\mathrm{a}}$, calculated from the least-squares fit to the points is $0.13 \mathrm{eV}$. If we plot the $Z^{\prime \prime}(\omega, T)$ data in the scaled coordinates, i.e. $Z^{\prime \prime}(\omega, T) / Z_{\max }^{\prime \prime}$ and $\log \left(\omega / \omega_{\mathrm{m}}\right)$, where $\omega_{\mathrm{m}}$ corresponds to the frequency of the peak value of $Z^{\prime \prime}$ in the $Z^{\prime \prime}$ versus $\log \omega$ plots, the entire data of imaginary part of impedance can collapse into one master curve as shown in figure 6 . Thus the scaling behaviour of $Z^{\prime \prime}$ clearly indicates that the relaxation mechanism is nearly temperature independent. 
The complex impedence plots of HCT at two temperatures $100^{\circ} \mathrm{C}$ and $150^{\circ} \mathrm{C}$ are shown in figure 7 . The values of resistance, $R$ and capacitance, $C$, can be obtained by an equivalent circuit of one parallel resistance-capacitance (RC) element. This RC element gives rise to one semicircular arc on the complex plane plot, representing the grain effect. The equivalent electrical equations for grain are

$$
\begin{aligned}
& Z^{\prime}=R+\frac{R_{\mathrm{g}}}{1+\left(\omega R_{\mathrm{g}} C_{\mathrm{g}}\right)^{2}}, \\
& Z^{\prime \prime}=R_{\mathrm{g}}\left[\frac{\omega R_{\mathrm{g}} C_{\mathrm{g}}}{1+\left(\omega R_{\mathrm{g}} C_{\mathrm{g}}\right)^{2}}\right],
\end{aligned}
$$

where $C_{\mathrm{g}}$ and $R_{\mathrm{g}}$ are the grain capacitance and grain resistance, respectively. We have fitted the experimental data using these expressions and the best fit of data, at $100^{\circ} \mathrm{C}$ with $R_{\mathrm{g}}=11.90 \mathrm{M} \Omega$ and $C_{\mathrm{g}}=1.75 \times 10^{-10} \mathrm{~F}$ and at $150^{\circ} \mathrm{C}$ with $R_{\mathrm{g}}=10.45 \mathrm{M} \Omega$ and $C_{\mathrm{g}}=1.99 \times 10^{-10} \mathrm{~F}$, are shown by solid line in figure 7 .

The angular frequency dependence of real part of electric modulus $\left(M^{\prime}\right)$ and imaginary parts $\left(M^{\prime \prime}\right)$ of HCT as a function of temperature is depicted in figure 8. From Figure $8(\mathrm{a})$, it is clear that $M^{\prime}(\omega)$ shows a dispersion behaviour tending towards $M_{\infty}\left(M_{\infty}=\right.$ the asymptotic value $M^{\prime}(\omega)$ at higher frequencies), while $M^{\prime \prime}(\omega)$ exhibits a maximum $\left(M_{\max }^{\prime \prime}\right)$ centred at the dispersion region of $M^{\prime}(\omega)$. The peaks developed in the values of $M^{\prime \prime}$ indicate a relaxation process (figure $8(\mathrm{~b})$ ). It may be noted that the position of the peak maximum $M_{\max }^{\prime \prime}$ shifts to higher frequencies as the temperature is increased showing the thermally activated nature of the relaxation time. The frequency region below peak maximum determines the range in which charge carriers are mobiles on long range distances and above peak maximum, the carriers are confined to potential wells being mobile on short distances. The frequency $\omega_{\mathrm{m}}$ corresponding to $M_{\max }^{\prime \prime}$ gives the most probable relaxation time $\tau_{\mathrm{m}}$ from the condition, $\omega_{\mathrm{m}} \tau_{\mathrm{m}}=1$.

The temperature dependence of the most probable relaxation time as shown in the inset of figure 9 also obeys the Arrhenius relation and the corresponding activation energy, $0.13 \mathrm{eV}$, for relaxation is found to be identical to the activation energy, $E_{\mathrm{a}}(=0.13 \mathrm{eV})$ for $Z^{\prime \prime}$. The activation energy values for electric modulus $(=0 \cdot 13 \mathrm{eV})$ and for d.c. conductivity $(=0.13 \mathrm{eV})$ are identical, indicating a hopping mechanism in HCT compound. The scaling behaviour of $M^{\prime \prime}$ i.e. $M^{\prime \prime} / M_{\max }^{\prime \prime}$ versus $\log \left(\omega / \omega_{\max }\right)$ plot of HCT at various

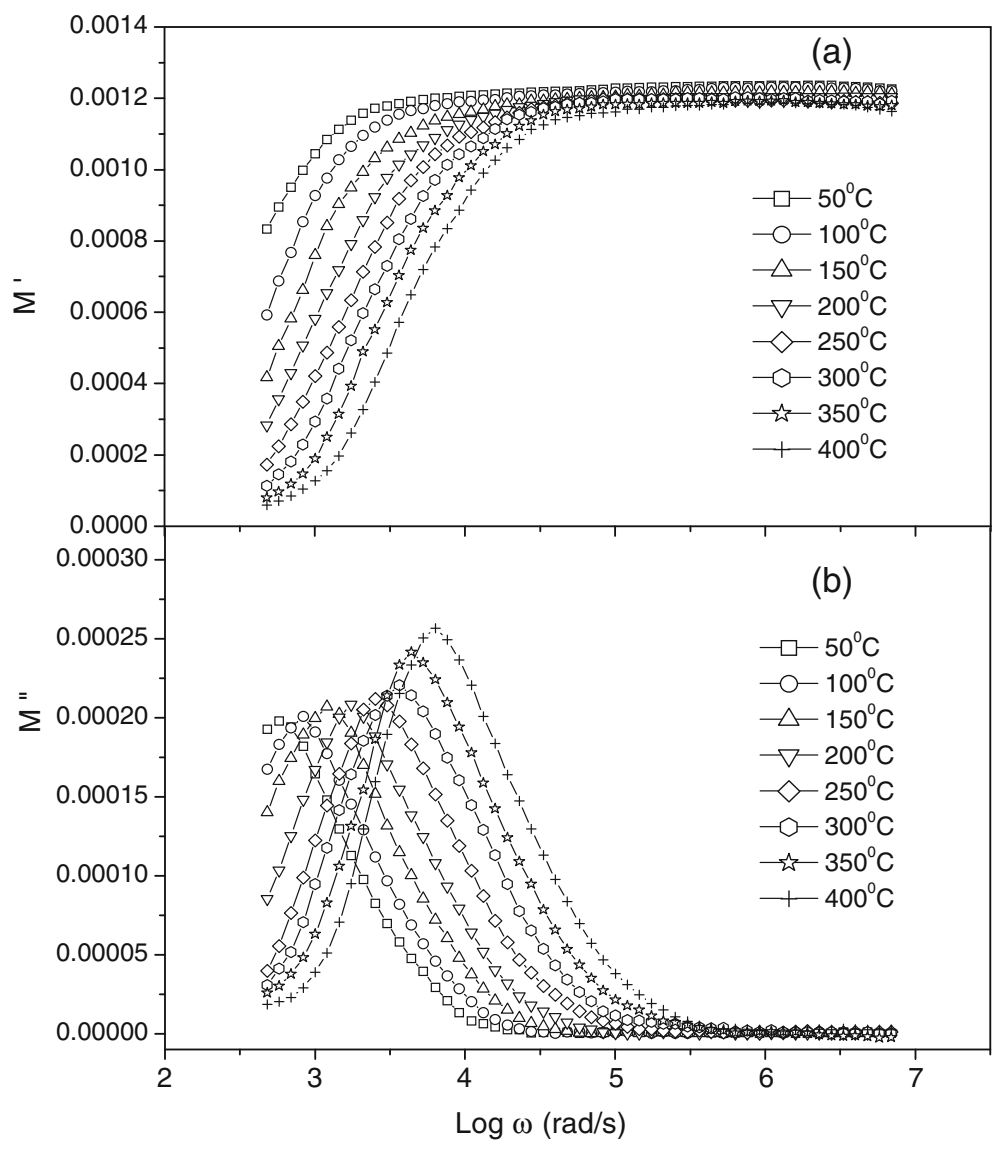

Figure 8. Frequency dependence of (a) $M^{\prime}$ and (b) $M^{\prime \prime}$ of $\mathrm{Ho}_{2} \mathrm{CdTiO}_{6}$ at various temperatures. 


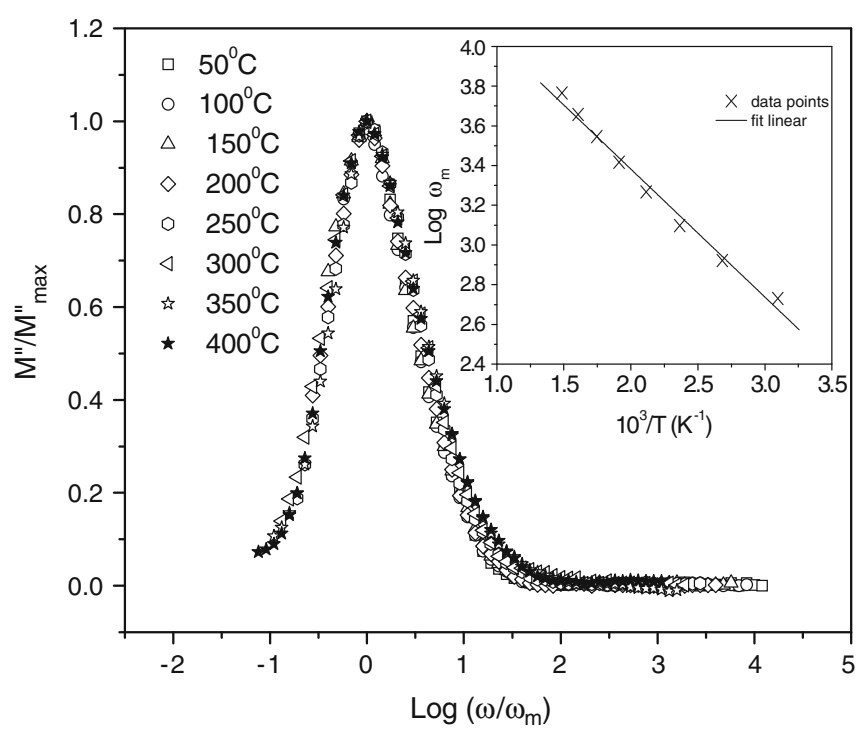

Figure 9. Scaling behaviour of $M^{\prime \prime}$ at various temperatures for $\mathrm{Ho}_{2} \mathrm{CdTiO}_{6}$. The temperature dependence of the most probable relaxation frequency obtained from the frequency-dependent plot of $M^{\prime \prime}$ is shown in the inset where the symbols are the experimental points and the solid line is the least-squares straight line fit.

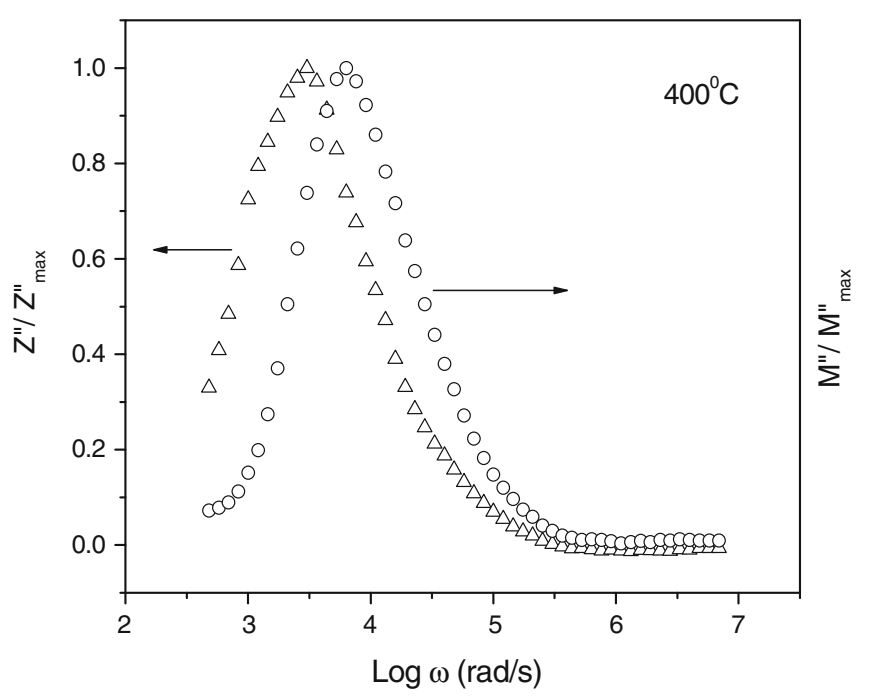

Figure 10. Frequency dependence of normalized peaks $Z^{\prime \prime} / Z_{\max }^{\prime \prime}$ and $M^{\prime \prime} / M_{\max }^{\prime \prime}$ for $\mathrm{Ho}_{2} \mathrm{CdTiO}_{6}$ at $400^{\circ} \mathrm{C}$.

temperatures is shown in figure 9. The overlapping of the curves for all the temperatures indicates that the dynamical processes are nearly temperature independent. Further in description of experimental data, the variation of normalized parameters $Z^{\prime \prime} / Z_{\max }^{\prime \prime}$ and $M^{\prime \prime} / M_{\max }^{\prime \prime}$ as a function of logarithmic frequency measured at $400^{\circ} \mathrm{C}$ for HCT is plotted in figure 10. Comparison with the impedance and electrical modulus data allow the determination of the bulk response in terms of localized (defect relaxation) or non-localized conduction (ionic or electronic conductivity) (Gerhardt 1994).
The Debye model is related to an ideal frequency response of localized relaxation. In reality, the non-localized process is dominated at low frequencies. In the absence of interfacial effects, the non-localized conductivity is known as the d.c. conductivity. From figure 10 it is obvious that the position of the peak in the $Z^{\prime \prime} / Z_{\max }^{\prime \prime}$ is shifted to a lower frequency region in relation to the $M^{\prime \prime} / M_{\max }^{\prime \prime}$ peak. It is possible to determine the type of dielectric response by inspection of the magnitude of overlapping between the peaks of both $Z^{\prime \prime}(\omega)$ and $M^{\prime \prime}(\omega)$. The overlapping peak position of $Z^{\prime \prime} / Z_{\max }^{\prime \prime}$ and $M^{\prime \prime} / M_{\max }^{\prime \prime}$ curves is evidence of delocalized or long range relaxation (Gerhardt 1994). Figure 10 shows that $Z^{\prime \prime} / Z_{\max }^{\prime \prime}$ and $M^{\prime \prime} / M_{\max }^{\prime \prime}$ do not overlap but are very close, suggesting the components from both long-range and localized relaxation.

\section{Conclusions}

The frequency-dependent dielectric dispersion of $\mathrm{Ho}_{2} \mathrm{CdTiO}_{6}$ ceramic synthesized by the solid-state reaction technique is investigated in the temperature range $50-400^{\circ} \mathrm{C}$ and in the frequency range $75 \mathrm{~Hz}-1 \mathrm{MHz}$ for the first time. The increasing dielectric constant and dielectric loss with decreasing frequency is attributed to the conductivity which is directly related to an increase in mobility of localized charge carriers. The frequency-dependent maxima of the imaginary part of impedance are found to obey Arrhenius law with an activation energy of $0.13 \mathrm{eV}$. Analysis of the real and imaginary parts of complex impedance with frequency were preferred assuming a distribution of relaxation times as confirmed by Cole-Cole plot as well as the scaling behaviour of impedance spectra. The scaling behaviour of the imaginary part of impedance spectra suggests that the distribution of relaxation times is temperature independent. The frequency-dependent electrical data are also analysed in the framework of the conductivity and modulus formalisms. The activation energy values for electric modulus $(=0.13 \mathrm{eV})$ and for d.c. conductivity $(=0.13 \mathrm{eV})$ are identical, indicating a hopping mechanism in HCT compound.

\section{Acknowledgement}

This work is financially supported by the Department of Science and Technology, New Delhi, under grant no. SR/S2/CMP-01/2008.

\section{References}

Anderson M T, Greenwood K B, Tailor G A and Poeppelmeier K R 1993 Prog. Solid State Chem. 22197

Bharti C and Sinha T P 2010 Solid State Sci. 12498

Bidault O, Goux P, Kchikech M, Belkaoumi M and Maglione M 1994 Phys. Rev. B 497868

Claeson T, Boyce J B, Bridges F, Gebale T H, Remeika J M and Sleight A W 1989 J. Phys. C 162-164544 
Cole K S and Cole R H 1941 J. Chem. Phys. 191484

DeMarco M 2000 Phys. Rev. B62 14301

Dutta A, Sinha T P and Shannigrahi S 2010 Jpn. J. Appl. Phys. 49 061504

Gerhardt R 1994 J. Phys. Chem. Solids 551491

Goodenough J B 1955 Phys. Rev. 100564

Hewat A W 1973 J. Phys. C6 1074

Kobayashi K I, Kimura T, Sawada H, Terakura K and Tokura Y 1998 Nature (London) 395677

Kumar R, Tomy C V, Nagarajan R, Paulose P L and Malik S K 2009 Physica B $\mathbf{4 0 4} 2369$

Kyritsis A, Pissis P and Grammatikakis J 1995 J. Polym. Sci. Polym. Phys. 331737
Lucuta P G, Constantinescu F and Barb D 1985 J. Am Ceram. Soc. 68533

Mitchell R H 2002 Perovskite: Modern and ancient (Ont, Canada: Almaz Press)

Raveau B 2007 Prog. Solid State Chem. 35171

Saines P J, Kennedy B J and Elcombe M M 2007 Solid State Chem. 180401

Sleight A W, Gillson J L and Bierdstedt P E 1993 Solid State Commun. 88841

Vijaykumar C, Kumar H P, Kavitha V T, Solomon S, Thomas J K, Wariar P R S and Koshy J 2009 J. Alloy Compd $\mathbf{4 7 5} 778$

Zhao F, Yue Z, Gui Z and Li L 2005 Jap. J. Appl. Phys. 44 8066 